\title{
Malignant Skin Tumor Misdiagnosed as a Benign Skin Lesion
}

\author{
So-Min Hwang, \\ Hao-Ching Pan, \\ Min-Kyu Hwang, \\ Min-Wook Kim, \\ Jong-Seo Lee \\ Aesthetic, Plastic and Reconstructive Surgery \\ Center
}

No potential conflict of interest relevant to this article was reported.

\begin{abstract}
Despite the fact that benign skin lesions can undergo malignant transformation, the necessity and timing of the surgical resection have yet to be established. In this study, we analyse three cases of benign-appearing skin lesions, which were found to be carcinomatous on histologic examination and review the literature regarding the importance of prophylactic removal of benign-appearing skin lesion. The first and second cases were female patients wishing for cosmetic surgery. The first patient had a benign-appearing lesion on dorsum nasi, and the second patient had an inconspicuous lesion right along the right nasolabial fold. The third patient was a middle-aged male with a pigmented lesion on the left cheek, who presented to the clinic only after having met the operating surgeon through an acquaintance outside the hospital setting. All of the lesions were suspected to be of benign nature and were excised for cosmesis only. However, histologic examination of these lesions showed that the first two tumors were basal cell carcinoma with the last tumor being squamouse cell carcinoma. Thus, it is considered that removal of benign like skin lesion will result in good prognosis of patients scheduled to undergo other surgery.
\end{abstract}

Keywords: Skin neoplasms / Face / Nevus

\section{INTRODUCTION}

In any given patient, the skin may contain various innate or acquired skin conditions. In general, most are common skin conditions with benign histology. However, the prevalence is high for skin lesions, with the majority of people having at least one type of pigmented skin disease. Despite the low rate of malignant transformation, the incidence of malignant skin tumors is high because the benign precursors are so highly prevalent. Because of this, skin cancers are known to be one of the most common carcinomas in the US [1].

Malignant skin tumors are distinguished between non-melanoma skin cancer (NMSC) and malignant melanoma. Despite

\section{Correspondence: So-Min Hwang}

Aesthetic, Plastic and Reconstructive Surgery Center, Good Moonhwa Hospital, 119, Beomil-ro, Dong-gu, Busan 48735, Korea

E-mail: sominhwang@hanmail.net

Received January 11, 2016 / Revised March 2, 2016 / Accepted April 16, 2016 the fact that benign skin lesions can undergo malignant transformation, the necessity and timing of the surgical resection have yet to be established [2]. In this study, we analyse three cases of benign-appearing skin lesions, which were found to be carcinomatous on histologic examination and review the literature regarding the importance of prophylactic removal of benignappearing skin lesion.

\section{CASE REPORTS}

This study was conducted via chart review of 3 patients visiting aesthetic plastic surgery clinic between August 2013 and January 2014. All three patients had presented with clinical issue unrelated to skin lesions.

The first patient was a 68-year-old woman who presented with sagging of upper and lower eyelids as a chief complaint, and was scheduled to undergo blepharoplasty. During examination, a $1 \times 1$ 
$\mathrm{cm}$ tumor was observed on the dorsum nasi, which the patient reported to have first noticed 10 years before. Though the tumor appeared to be benign, the patient was offered resection as an aesthetic option at the time of blepharoplasty operation. The patient consented, and the tumor with $2 \mathrm{~mm}$ resection margin resection and blepharoplasty were performed simultaneously (Fig. 1).

The second patient was a 59-year-old woman who presented with sagging of the upper eyelid and nasolabial folds, and was scheduled to undergo blepharoplasty of the upper eyelid and a filler injection. A $0.5 \times 0.5 \mathrm{~cm}$ tumor was present on the right nasolabial fold, which the patient had first noted 15 years prior. The patient consented to and underwent resection of the lesion with $1 \mathrm{~mm}$ resection margin for aesthetic purposes, along with blepharoplasty and filler injection (Fig. 2).

The third patient was a 57-year-old man who encountered accidentally in a private gathering, and a tumor measuring $1.5 \times 1.0$ $\mathrm{cm}$ in size, which had developed 5 years before was observed in the malar area. The tumor resection was recommended for aesthetic purpose, and thus the patient visited our hospital to undergo tumor resection with $2 \mathrm{~mm}$ resection margin (Fig. 3).

Although all 3 lesions were suspected to be benign and were resected for aesthetic purposes, histologic examination revealed the first two lesions to be basal cell carcinoma (Fig. 4) and the third lesion to be squamous cell carcinoma (Fig. 5). Fortunately,
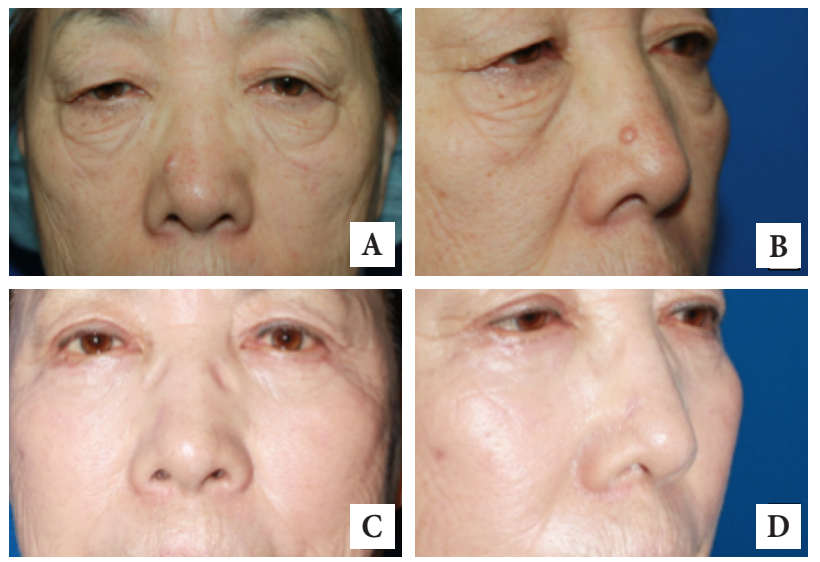

Fig. 1. The first is a 68-year-old female who had presented with aging periorbital soft tissue changes. The patient was offered and consented to the resection of the 1-cm mass on nasal dorsum, which had developed over a decade. (A, B) In the preoperative photograph, the mass is very obvious but benign-appearing. (C, D) Postoperative appearance at 6 months is satisfactory. the margins were free of cancer cells. Hematologic and chest radiological examinations of these 3 patients showed no abnormal findings, with unremarkable review of systems.

The first and second patients with confirmed diagnosis of basal cell carcinomas were followed in the outpatient clinic for more than a year without local recurrence. The third patient with squamous cell carcinoma was followed at another hospital and remain free of recurrence at the 18-month follow-up.
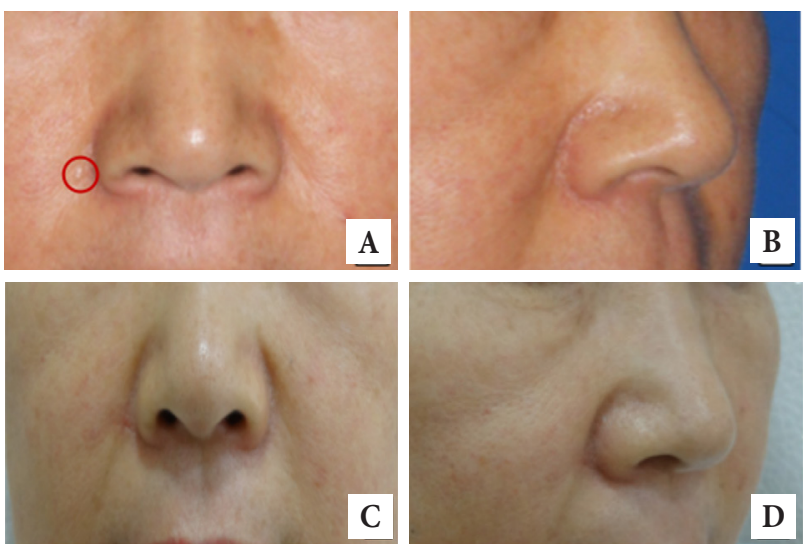

Fig. 2. The second patient is a 59-year-old female who presented with sagging upper eyelids and deepening of the nasolabial fold. Facial examination revealed a small palpable nodule along the right nasolabial fold (red circle), which the patient believed to have developed over a 15 year period. This nodule was resected at the time of upper blepharoplasty and filler injection. (A, B) In the preoperative photograph, the mass is not extremely conspicuous. (C, D) Postoperative appearance at 4 months is satisfactory.
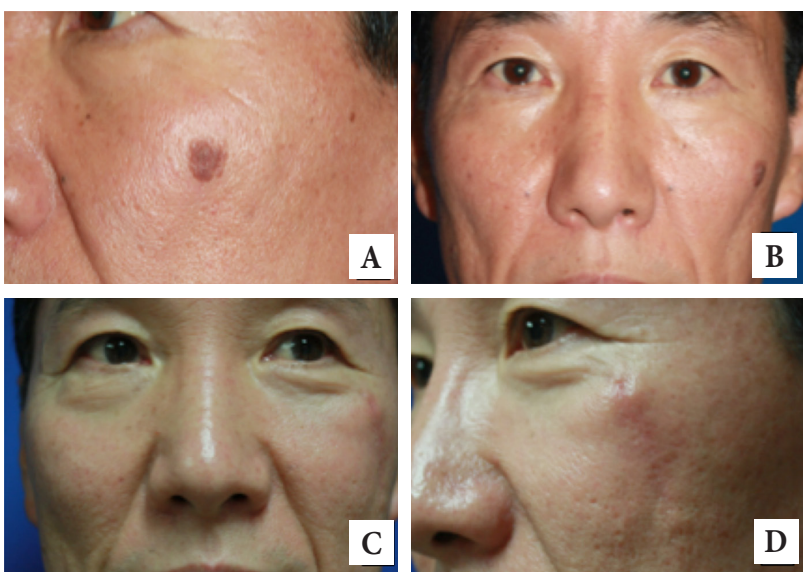

Fig. 3. The third patient is a 57-year-old male who presented with a $1.5-\mathrm{cm}$ pigmented lesion on the left cheek. The lesion had developed over the past 5 years, but the patient did not seek medical attention until meeting the operating surgeon through an acquaintance outside the hospital setting. (A, B) In the preoperative photograph, the cheek lesion appears benign with uniform pigmentation and normal border. (C, D) Postoperative scar is healing well at 3 months. 

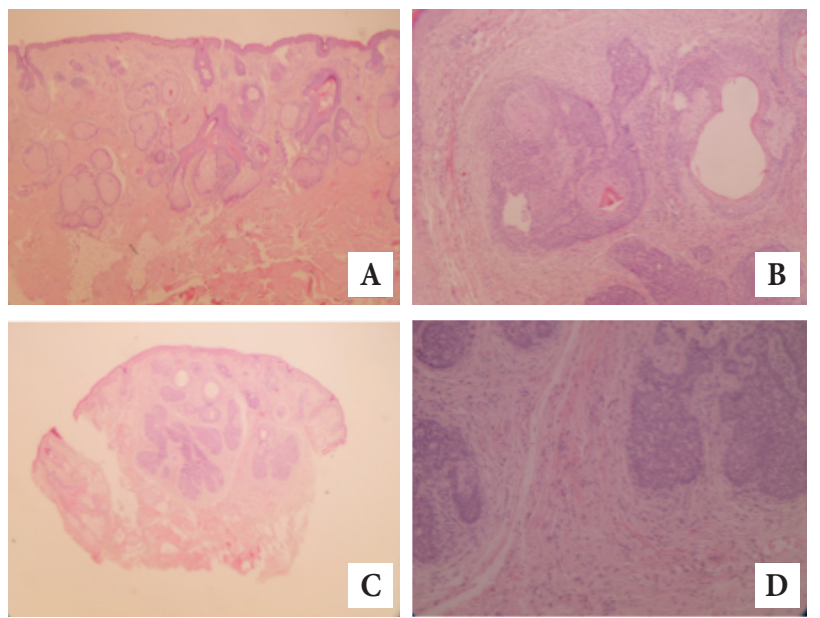

Fig. 4. Histologic examination of the skin lesions from first and second cases. (A) Hyperchromatism is observed in the histologic examination of first patient (H\&E, $\times 40)$. (B) Scant cytoplasm and tumor cell nest is observed in the histologic examination of first patient $(\mathrm{H} \& \mathrm{E}, \times 200)$. (C) Hyperchromatism is observed in the histologic examination of second patient $(\mathrm{H} \& \mathrm{E}, \times 40)$. (D) Scant cytoplasm and tumor cell nest is observed in the histologic examination of second patient $(\mathrm{H} \& \mathrm{E}, \times 200)$.
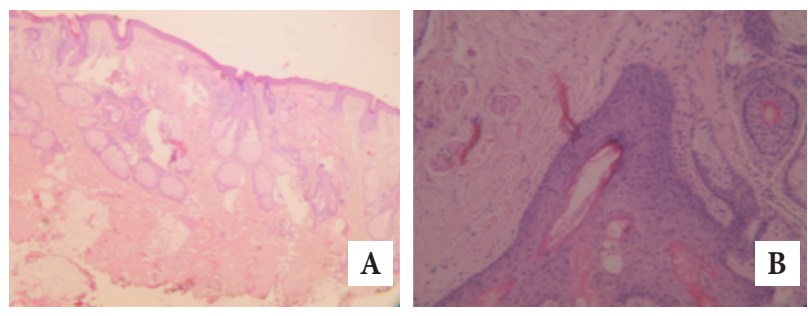

Fig. 5. Histologic examination of the skin lesion from the third patient. (A) Abundant cytoplasm and extracellular keratin pearls observed (H\&E, $\times 40)$. (B) Pleomorphism, hyperchromatism observed (H\&E, ×200).

\section{DISCUSSION}

Skin cancers are very common, occurring in 1 in 6 people throughout lifetime in the US. Generally, cutaneous cancers are distinguished between NMSC and malignant melanoma. The most common type of NMSC is basal cell carcinoma, which occurs in 212 to 253 and 407 to 485 per 100,000 females and males, respectively. The lifetime prevalence is $28 \%$ to $33 \%[3,4]$.

Squamous cell carcinomas are known to occur in 26 to 59 and 81 to 136 per 100,000 females and males, respectively, with lifetime prevalence of $7 \%$ to $11 \%$ [4].

Anatomically, the high incidence of NMSC in the head and neck region is believed to be due to relatively high exposure to incident sunlight unto the face [5]. The head and neck regions account for $85 \%$ of BCC, with the nose accounting for $25 \%$ to $30 \%[6]$.

In the US population, malignant melanoma has the 8th highest prevalence. In general, the prevalence of malignant melanoma is $1 / 20$ th of the prevalence for NMSC, and is highly dependent on skin color. While the prevalence among African-Americans is less than 1 in 100,000 person per year, malignant melanoma occurs among Caucasians at 50 in 100,000 person per year $[3,4]$. The lifetime risk is $1.94 \%$ in males and $1.3 \%$ in females, and the mortality is $0.35 \%$ in males and $0.2 \%$ in females [3]. Similarly to NMSC, the prevalence for melanoma has increased by $3 \%$ to $7 \%$ due to the increase in outdoor activities and exposure to ultraviolet rays, changes in preferred clothing, prolonged average life span, and reductions in the ozone layer. Although the prevalence is lower for melanoma than for other types of skin cancer, melanoma is a more dangerous disease due to the possibility of metastasis and higher rates of mortality $[1,3,4]$.

As the prevalence increases for various skin cancers, the necessity of screening tests is also increasing. However, diagnosis of skin cancer is generally performed with the naked eye, which causes frequent misdiagnosis. Accordingly, there is a possibility of over-treatment or delayed treatment, and realistically, observation of skin of the whole body is difficult. For these reasons, screening tests are not performed.

Nevus is a representative example. It is a benign congenital condition of the skin of hamartomatous histology. The lesions are concentrated in the head and neck, with 63\% observed on the scalp and $34.7 \%$ on the face and neck [7]. According to epidemiologic studies, malignant transformation occurs in less than $1 \%$ of nevus sebaceous, and most cases of malignant transformations occur after adolescence. Because of the low rate of malignant transformation and the lack of need for early follow up, the necessity and timing of the prophylactic resection are still under debate $[2,7,8]$.

Late presentation is the cause for greater than $70 \%$ of delayed diagnoses. Patients delay seeking medical care for a number of reasons, including fear of the diagnosis and treatment process, absence of family history of skin cancer, and asymptomatic le- 
sions. Misdiagnosis can also cause delay in diagnosis. In many instances, superficial BCC may be misdiagnosed as psoriasis, lichen planus, or lichenoid keratosis. Pigmented BCC have been misdiagnosed as melanocytic nevus or seborrheic keratosis SCC may be misdiagnosed as actinic keratosis, keratoacanthoma and bowen disease [9]. The skin lesion in our third case also can be misdiagnosed as seborrheic keratosis and warts..

Skin lesions should be resected for characteristics of malignant transformation, such as asymmetry, border irregularity, color variability, diameter of $6 \mathrm{~mm}$ or more, rapid growth, shiny and pearly papule, elevated nodule, or telangiectasia $[3,6]$. Regarding the margin of skin lesion for resection, approaches to prophylactic resection for non-cancerous skin lesions and curative resection for cancerous skin lesions are different in our hospital, and the resection margin for non-cancerous skin lesions is set to be 1-2 mm depending on the site of lesion and skin redundancy. For the cancerous skin lesion, punch biopsy is performed before resection, and 5-30 $\mathrm{mm}$ of resection margin is planned depending on the cancer type and differentiation [10]. Subsequently, Mohs surgery is performed for resection, and the resected sample undergoes frozen biopsy to determine whether resection is clear. If it is confirmed to be clear, closure is performed. If there is a possibility of residual lesions in the patient having undergone prophylactic resection due to incomplete resection margin, re-excision is performed in case that the unclear site is the deep surgical margin of the sample, the location of lesion includes critical midfacial site, defect is repaired using skin flap or skin graft after excision, or aggressive histological subtype is observed in biopsy [11].

Regarding the misdiagnosis of skin lesions, Heal et al. [12] suggested that the positive predictive value according to each type of skin lesions was not high, shown to be $72 \%$ for basal cell carcinoma, $49.9 \%$ for squamous cell carcinoma, and 33\% for cutaneous melanoma. Therefore, due to the nature of skin cancer that should be diagnosed only with the patient's history and gross lesion appearance, it is difficult to make an accurate diag- nosis, and subsequently it is common that benign lesions are diagnosed as malicious or vice versa.

Hence, considering the rare cases of benign-looking lesions developing to skin cancer along with the cases in this study, it is suggested that recommendation of resection of skin lesions will lead to good outcomes in a patient scheduled for surgery for aesthetic or other purposes.

\section{REFERENCES}

1. Dilek N, Bedir R, Yuksel D, Ataseven A. A case of basal cell carcinoma secondary to nevus sebaceous. Int J Case Rep Imag 2014;5:277-80.

2. Enei ML, Paschoal FM, Valdes G, Valdes R. Basal cell carcinoma appearing in a facial nevus sebaceous of Jadassohn: dermoscopic features. An Bras Dermatol 2012;87:640-2.

3. Rosen H, Schmidt B, Lam HP, Meara JG, Labow BI. Management of nevus sebaceous and the risk of Basal cell carcinoma: an 18-year review. Pediatr Dermatol 2009;26:676-81.

4. Christenson LJ, Borrowman TA, Vachon CM, Tollefson MM, Otley CC, Weaver AL, et al. Incidence of basal cell and squamous cell carcinomas in a population younger than 40 years. JAMA 2005;294:68190 .

5. Linden KG. Screening and early detection of skin cancer. Curr Oncol Rep 2004;6:491-6.

6. U.S. Preventive Services Task Force. Screening for skin cancer: U.S. Preventive Services Task Force recommendation statement. Ann Intern Med 2009;150:188-93.

7. Diepgen TL, Mahler V. The epidemiology of skin cancer. Br J Dermatol 2002;146 Suppl 61:1-6.

8. Wiznia LE, Shanley KM, Federman DG. Advanced, neglected basal cell carcinoma. South Med J 2014;107:242-5.

9. Karynne OD, John KG, David JL. Epithelial precancerous lesions. In: Fitzpatrick TB, Goldsmith LA, editors. Fitzpatrick's dermatology in general medicine. 8th ed. New York: McGrawHill; 2012. p.1261-90.

10. Ogawa R. Benign and malignant nonmelanocytic tumors of the skin and doft Tissue. In: Neligan PC, Gurtner GC, editors. Plastic Surgery. 3rd ed. Seattle: Elsevier; 2013.p.717-42.

11. Telfer NR, Colver GB, Morton CA; British Association of Dermatologists. Guidelines for the management of basal cell carcinoma. $\mathrm{Br} \mathrm{J}$ Dermatol 2008;159:35-48.

12. Heal CF, Raasch BA, Buettner PG, Weedon D. Accuracy of clinical diagnosis of skin lesions. Br J Dermatol 2008;159:661-8. 